A N N A L E S Annales de Bretagne et des Pays de l'Ouest

\title{
L'enquête de 1665 sur le littoral provençal : un état des lieux pour l'État royal
}

The 1665 survey of the Provençal coast: an inventory for the Royal State

\section{Gilbert Buti}

\section{OpenEdition}

1 Journals

\section{Édition électronique}

URL : https://journals.openedition.org/abpo/4632

DOI : $10.4000 / a b p o .4632$

ISBN : 978-2-7535-7968-2

ISSN : 2108-6443

\section{Éditeur}

Presses universitaires de Rennes

Édition imprimée

Date de publication : 20 novembre 2019

Pagination : 78-99

ISBN : 978-2-7535-7966-8

ISSN : 0399-0826

\section{Référence électronique}

Gilbert Buti, «L'enquête de 1665 sur le littoral provençal : un état des lieux pour l'État royal », Annales de Bretagne et des Pays de l'Ouest [En ligne], 126-3 | 2019, mis en ligne le 01 janvier 2022, consulté le 08 janvier 2022. URL : http://journals.openedition.org/abpo/4632 ; DOI : https://doi.org/10.4000/abpo. 4632 


\title{
L'enquête de 1665 sur le littoral provençal : un état des lieux pour l'État royal
}

\author{
Gilbert BuTI \\ Professeur émérite d'histoire, Aix-Marseille Université, CNRS, TELEMMe, \\ Aix-en-Provence, France
}

De grandes enquêtes administratives ont été réalisées par les États européens du XIII ${ }^{\mathrm{e}}$ au XV $\mathrm{XV}^{\mathrm{e}}$ siècle afin de disposer de connaissances solides pour guider leur action. En usant de différents moyens de collecte, ils ont recueilli un abondant matériau documentaire de différentes natures auprès de multiples acteurs et témoins. Dans la mesure où " gouverner, c'est enquêter ", il s'agissait pour les promoteurs de ces opérations de déterminer les contours du domaine princier et d'en évaluer les ressources, voire de corriger et de réformer les pratiques des officiers ${ }^{1}$. Au reste, par leur rituel de mise en ordre et en liste, ces enquêtes ont pu exercer une fonction idéologique et restaurer un sentiment d'appartenance ou de fidélité. Mais le fonctionnement de la société, les dénombrements de la population et la description de la diversité culturelle étaient peu à l'ordre du jour.

À partir du XVII ${ }^{\mathrm{e}}$ siècle, en France, le pouvoir central a diligenté des enquêtes à finalité plus pratique pour engager certaines réformes liées au développement de la monarchie administrative et à l'activité croissante de ses bureaux ${ }^{2}$. Étroitement associées au fonctionnement de l'État, ces enquêtes étaient réservées à l'usage du commanditaire et devaient rester secrètes. Dénombrer les populations, faire l'inventaire des subsistances, enregistrer les prix, avec des arrière-pensées fiscales et militaires, figurent parmi les objectifs assignés à ces opérations. La propagation des idées mercantilistes, favorables au développement d'une politique maritime, et les conflits de la première moitié du XVII ${ }^{\mathrm{e}}$ siècle qui impliquent la mise en œuvre

1. PÉcout, Thierry (dir.), Quand gouverner, c'est enquêter. Les pratiques politiques de l'enquête princière, Occident, XIII ${ }^{e}$-XIV ${ }^{v}$ siècles, Paris, De Boccard, 2010.

2. Pour une vue globale on se reportera à ACERRA, Martine, BuTI, Gilbert, LLINARÈs, Sylviane, PfISTER, Christian, "Inspecter le littoral français à l'époque moderne " dans $L a$ maritimisation du monde, Paris, Presses universitaires de Paris-Sorbonne, 2016, p. 497-513. 
de flottes permanentes et de bases navales, rendent compte de cet intérêt nouveau pour l'État moderne en construction. Les enquêtes engagées par Richelieu puis Colbert sont des étapes essentielles de cette appropriation du domaine maritime et de la tutelle mise, par le pouvoir central, sur les rivages, les hommes, les ports et les navires.

Pour dresser un état des lieux de chacune des façades maritimes du royaume, Richelieu a attribué des missions d'inspection à des hommes de confiance : l'inspection des côtes du Ponant à Langlois et Leroux, chevalier d'Infreville, celle des côtes du Levant à Henri de Séguiran, seigneur de Bouc, premier président de la Cour des comptes, aides et finances de Provence. La mission accomplie par celui-ci, en 1633 et 1635, a surtout eu un objectif défensif : identifier les ports à entretenir et repérer des lieux à fortifier ou à aménager. Il s'agissait également " de faire dresser une vue figurée de toute la côte maritime afin que sur cette figure il [Richelieu] pût ordonner les fortifications nécessaires pour la défense du pays et empêcher la descente des ennemis ${ }^{3}$ ». Pour ce faire, Séguiran, accompagné d'un professeur de mathématiques (Jacques de Maretz) et de deux cartographes (Augier et Flour d'Arles), a visité une trentaine de localités en 65 jours et interrogé dans chacune d'elles " les plus apparents " venus à sa rencontre. La procédure est classique car déclenchée "d'en haut " et menée par des personnes spécialement détachées pour cela. Elle est exceptionnelle, car elle n'a pas été employée régulièrement par la monarchie pour les affaires maritimes, et elle est fragile dans la mesure où les renseignements recueillis, peu suivis d'effet, sont rapidement périmés.

Les deux grandes enquêtes royales suivantes sont à mettre à l'actif de l'administration de Colbert au début du gouvernement personnel de Louis XIV. La première, qui fait réponse à l'arrêt pris au Conseil royal des finances du 21 avril 1664, est confiée " aux officiers des amirautés et autres juges exerçant les causes maritimes en ce royaume ${ }^{4}$ ". Le roi leur demande de " faire une description, état ou inventaire général de tous les vaisseaux de quelques fabriques qu'ils puissent être, française ou étrangère, qui se trouveraient dans les ports, havres et rades [...] appartenant à des particuliers marchands, négociants ou autres sujets de sa dite Majesté ${ }^{5}$ ". L'enquête se déroule pendant un peu plus d'un mois et les réponses des amirautés remontent à Colbert qui a accompagné l'arrêt royal d'une série

3. Arch. dép. des Bouches-du-Rhône, IX B 2 - Brevet royal nommant, sur la présentation du cardinal de Richelieu, M. de Séguiran, premier président de la Cour des comptes de Provence, aux fonctions de lieutenant du cardinal en Provence pour le fait de la marine, 24 septembre 1632 , fo $252-253$.

4. Cette précision concerne la Bretagne, sans implantation d'amirauté avant 1691 et où ce sont le plus souvent les officiers de justice des présidiaux qui répondent aux exigences royales (MorineAU, Michel, «De l'efficacité de l'administration en France au début du règne de Louis XIV. À propos de l'Inventaire de la Marine de commerce de 1664 ", dans Histoire comparée de l'administration ( $I^{e}$-XVIII s.), Beihefte der Francia, t. 9, Munich, 1980, p. 26-32).

5 . Bibliothèque nationale de France (désormais BnF), département des manuscrits, 500 Colbert, $\mathrm{n}^{\circ} 199$. 
de questions précises. La moindre barque paraît avoir été recensée et les navires sont décrits avec plus ou moins de précision et de zèle ${ }^{6}$. Cet inventaire est complété l'année suivante à la suite de " diverses plaintes " faites au roi, selon lesquelles " les ports, havres, rades, baies et rivières de ce royaume deviennent de jour en jour moins sûrs et praticables et beaucoup plus difficiles soit pour l'entrée que la sortie ou pour la demeure et séjour desdits navires et autres bâtiments qui y arrivent ordinairement ${ }^{7}$ ".

C'est la conduite de cette enquête, qui s'inscrit également dans le projet de relèvement de la marine royale, que nous nous proposons de suivre à partir de quatre postes d'observation pris sur le littoral provençal, très peu soumis au phénomène des marées et où les administrateurs des ports comme les gens de mer signalent, de manière récurrente, les risques d'envasement des bassins portuaires. Ainsi en est-il de Martigues à Saint-Tropez en passant par Marseille et Toulon, ports d'inégale importance, n'occupant pas les mêmes types de site, n'étant pas soumis aux mêmes contraintes naturelles et présentant différentes orientations économiques ${ }^{8}$. Situé sur les rives de l'étang de Berre et réputé pour sa construction navale, Martigues est, au milieu du $\mathrm{XVII}^{\mathrm{e}}$ siècle, un port de pêche et de commerce actif, tourné surtout vers le bassin occidental de la Méditerranée. La pêche et plus encore le petit cabotage animent les activités du modeste bassin portuaire de Saint-Tropez, qui sert parfois de refuge à des galères battant divers pavillons. Situé au fond d'une grande rade, le port de Toulon ne comprend alors qu'une seule darse et n'est pas encore un véritable port de guerre, malgré les projets d'y établir, depuis Henri II et plus encore Richelieu, un arsenal. Le port de Marseille, qui occupe la calanque du Lacydon, est assurément le plus important de la France méridionale, et à sa fréquentation par des bâtiments de commerce se livrant surtout à un trafic méditerranéen, il faut ajouter la présence de galères imposée par le jeune Louis XIV ${ }^{9}$.

Les procès-verbaux, adressés par les responsables des amirautés de ces ports provençaux aux profils différents, invitent à suivre, au-delà des cadres définis par le pouvoir central, la diversité des modalités retenues localement pour répondre à des demandes précises, à identifier les acteurs mobilisés pour procéder aux opérations et à décrypter les réponses ou suggestions formulées.

\section{L'enquête de 1665 : procédure}

La police des ports et des havres a souvent donné lieu en France à une réglementation locale spécifique avec la perception de taxes et d'amendes

6. BuTI, Gilbert, « La flotte de commerce et de pêche de la France méditerranéenne à la fin du XvII siècle d'après les enquêtes de Colbert et Seignelay ", dans Tourville et les marines de son temps, Saint-Lô, Arch. dép. de la Manche, 2003, p. 49-90.

7. BnF, département des manuscrits, 500 Colbert, $\mathrm{n}^{\circ} 199$.

8. Martigues compte environ 16000 habitants, Saint-Tropez 2400, Toulon 18000 et Marseille 65000 à 68000 (avec le terroir)

9. ZYSBERG, André, Marseille au temps du Roi-Soleil, Marseille, Jeanne Laffitte, 2007. 
Figure 1 - De Martigues à Saint-Tropez

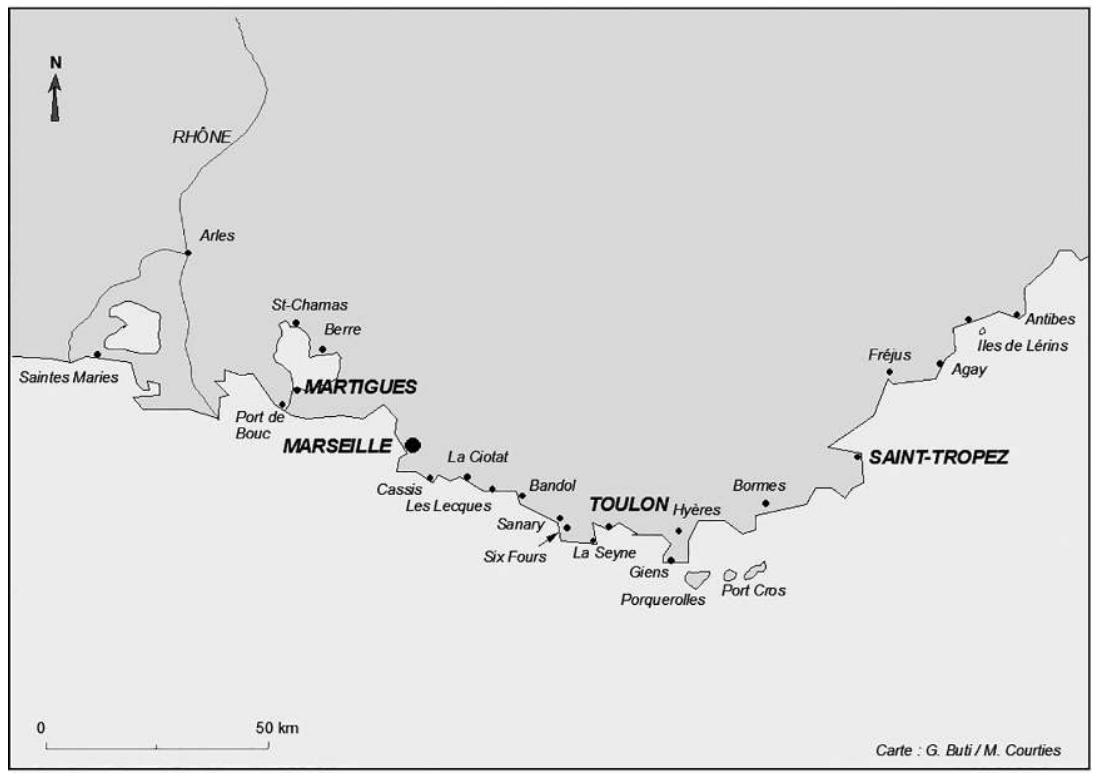

pour prévenir les risques d'envasement et préserver les résultats d'onéreux travaux de curage et de creusement. La question du délestage des navires, qui participe à cette dégradation, est abordée, au cours du XVII ${ }^{\mathrm{e}}$ siècle, notamment dans le code Michaud de 1629 qui condamne les contrevenants à une peine de confiscation de navires. Dans la mesure où la dégradation de l'outil portuaire viendrait " principalement du peu de soin qu'on a eu d'empêcher que les maîtres et patrons de navires déchargent le lest de leurs vaisseaux en des lieux assez éloignés des ports, rades et embouchures des rivières pour n'y pas causer de dommage ", l'objectif de l'arrêt de janvier 1665 est clair :

" les officiers des amirautés auront à visiter et faire sonder [...] la profondeur des havres, rades, embouchures du port et rivières dépendantes de leur juridiction, à dresser procès-verbal du bon ou mauvais état auquel ils se trouveront [...] et à faire enquête sommaire du dépérissement et particulièrement des lieux où les maîtres de navires français ou étrangers déchargent les lests."

Les modalités retenues pour mener cette opération sont semblables à celles de 1664 dans la mesure où un arrêt du Conseil du roi, en date du 24 janvier 1665, sollicite pour y répondre les 48 sièges d'amirauté et autres juridictions. Au lieu de confier l'exécution des opérations à des personnages mandatés, comme au temps de Richelieu, Colbert en charge directement les officiers des amirautés détenteurs de l'autorité royale en matière de justice maritime et habilités, de par leur résidence, à connaître les arma- 
teurs, les navires et les ports. Par ce choix il supprime un intermédiaire, à savoir l'envoyé qui doit se déplacer, renforce l'allégeance des amirautés à l'égard du pouvoir central et réduit, en principe, les délais de réponses.

Par ailleurs, sur place, les officiers des amirautés sont débarrassés d'une présence encombrante pouvant contrôler leur siège et se trouvent investis d'une autorité affirmée vis-à-vis de leurs justiciables car pouvant se prévaloir de la confiance du roi. Néanmoins, le succès de l'opération n'est pas acquis d'avance car l'amirauté de France possède une certaine autonomie et, au moment de l'enquête, Colbert n'a pas encore officiellement dans ses attributions le département de la Marine. Celui-ci est toujours rattaché au secrétariat d'État des Affaires étrangères, mais Lionne, qui en a la charge, ne siège pas au Conseil des finances où Colbert rapporte sur les affaires de la marine. La mise en route des opérations est donc prudente : elle démarre par un arrêt signé par Lionne, mais précise que les procès-verbaux seront " promptement envoyés à Monseigneur Colbert ${ }^{10}$ ". Il s'agit d'inspecter les havres et côtes de France, "plus ou moins recommandables ", selon des critères minutieusement définis par Colbert, au fil des 38 demandes d'informations portant notamment sur les dégradations nées du délestage dans les ports ${ }^{11}$. Accompagnée d'instructions très détaillées, la lettre circulaire est expédiée peu après la prise de décision et les réponses sont attendues dans les meilleurs délais en respectant, pour leur présentation, un modèle standard fourni par les bureaux centraux qui ont peut-être l'intention d'en composer, in fine, un recueil.

La demande ministérielle est enregistrée plus ou moins rapidement aux greffes des amirautés ${ }^{12}$. En Provence, les opérations, qui sont engagées entre quinze jours et trois semaines après cet enregistrement, durent de un à huit jours et donnent lieu à des procès-verbaux d'importance inégale, de trois folios (soit six pages) pour Marseille à treize pour Toulon, parfois accompagnés d'illustrations et d'un nombre variable de signatures.

10. Service historique de la Défense (ShD), Vincennes, ms SH 48. Instruction envoyée à tous lesdits officiers pour leur dire la manière de faire ladite visite afin de connaître les bonnes et mauvaises qualités des ports. Ce manuscrit a fait l'objet d'une approche globale par FlORA, Mathieu (master, sous la direction de Martine ACERRA, université de Nantes, 2011-2012).

11. Pour le lieu, doivent être observés la configuration naturelle, l'adaptation aux activités maritimes, la protection, le potentiel de création portuaire, les possibilités d'amélioration. Pour les hommes, sont ciblés les charpentiers, géographes, hydrographes, pilotes, calfats, canonniers, cordiers, matelots, et pour les activités ce sont tout naturellement la pêche, le commerce et la guerre. Quant aux ressources locales, il est recommandé de noter la présence d'eau douce, de bois de chêne, de hêtre, de chanvre, lest, vin, blé, fer et victuailles pour les vaisseaux.

12. Le siège d'amirauté de Saint-Tropez, mentionné dans le procès-verbal de 1665 mais non dans celui de 1664, a été créé en 1649 par dédoublement de l'amirauté de Fréjus. 


\begin{tabular}{|l|c|c|c|c|c|c|}
\hline & $\begin{array}{c}\text { Enregis- } \\
\text { trement }\end{array}$ & $\begin{array}{c}\text { Début } \\
\text { des opé- } \\
\text { rations }\end{array}$ & $\begin{array}{c}\text { Fin des } \\
\text { opéra- } \\
\text { tions }\end{array}$ & $\begin{array}{c}\text { Durée } \\
\text { des opé- } \\
\text { rations } \\
\text { (jour) }\end{array}$ & $\begin{array}{c}\text { Nombre } \\
\text { de } \\
\text { folios du } \\
\text { procès- } \\
\text { verbal }\end{array}$ & $\begin{array}{c}\text { Signa- } \\
\text { tures }\end{array}$ \\
\hline Martigues & $?$ & 22 mai & 22 mai & 1 & 5 & 12 \\
\hline Marseille & 17 mars & 8 avril & 10 avril & 3 & 3 & 3 \\
\hline Toulon & 20 mai & 10 juin & 17 juin & 8 & 13 & 1 \\
\hline Saint-Tropez & $?$ & 30 avril & 30 avril & 1 & 5 & 1 \\
\hline
\end{tabular}

Le lieu où débute l'enquête est clairement indiqué pour Marseille (maison d'habitation de Valbelle où fut accueillie, quelques mois plus tôt, la mère du jeune Louis XIV) et pour Toulon (maison de Ricard, lieutenant de l'amirauté) mais reste en revanche indéterminé à Martigues et Saint-Tropez. Les procès-verbaux sont naturellement établis par les greffiers des amirautés (François Bayon à Marseille, Gairoard à Toulon, Massel à Saint-Tropez et François Pons, également notaire royal, à Martigues) sous l'autorité des responsables locaux : conseillers du roi et lieutenants généraux de l'amirauté, procureurs du roi. À Marseille, les opérations sont placées sous la responsabilité de Léon de Valbelle (1634-1691), " conseiller du roi et lieutenant général des mers du Levant en l'amirauté ", issu d'une puissante famille provençale qui a tenu la ville pendant la Fonde. Ce sont également les représentants d'une vieille famille que l'on trouve à Toulon, à savoir les Ricard, François et Vincent (père et fils ou frères?), le premier en qualité de lieutenant "principal " ou " général ", l'autre de " lieutenant particulier " remplaçant le premier " empêché " pour effectuer la visite du port, en compagnie de Jassinthe ou Jachinthe Ricard. À Martigues, le lieutenant de l'amirauté n'est pas nommé, l'opération se déroulant sous la responsabilité de Pierre Romey " exerçant comme plus ancien de la juridiction de l'amirauté au siège de cette ville de Martigues en place de Monsieur le lieutenant audit siège ".

Les procès-verbaux de Martigues, Marseille et Saint-Tropez décrivent les opérations après un bref rappel de la demande royale ou du résumé de celle-ci, alors qu'à Toulon le procès-verbal reproduit intégralement l'arrêt du Conseil et l'accompagne des principes qui seront suivis pour son application à savoir :

"Visiter et faire sonder par personnes capables la profondeur du port, rade de cette ville, dresser procès verbal du bon ou mauvais état auquel il se trouvera, entendre les plus anciens experts de cette ville sur l'état auquel était le dit port et rade et la différence de celui auquel il est à présent, faire enquête sommaire du dépérissement et particulièrement des lieux où les maîtres de navires français ou étrangers déchargent les lests et si lesdites décharges n'ont point rempli l'embouchure du port et rade..."

Cet exposé des modalités, que le lieutenant de l'amirauté entend faire suivre, est adressé au roi le 10 juin 1665, soit la veille de l'inspection. 
Le choix des "personnes intelligentes et capables " ou " experts ", selon les termes employés dans l'arrêt du 24 janvier 1665 et repris par les procès-verbaux, est laissé à l'initiative des responsables locaux. Pas plus que leur " qualité ", le pouvoir central n'en fixe le nombre. À Martigues, ce sont douze personnes qui sont nommées par Pierre Romey, à Marseille, Valbelle en désigne huit, à Toulon, le procureur du roi et le lieutenant de l'amirauté en choisissent huit, et deux seulement sont désignés à Saint-Tropez.

\section{De complexes et inégales enquêtes de terrain}

Afin d'éviter les « brouillages " qui pourraient se produire si l'on menait de front l'analyse des opérations conduites dans les différents ports, leur présentation séparée permettra d'en pointer la richesse et les spécificités.

Parmi les "personnes intelligentes et capables " qui interviennent à Martigues figure le premier consul, Simon Peignier ${ }^{13}$. Le premier magistrat de la cité - une présence qui ne se retrouve pas dans les autres ports étudiés - est accompagné du greffier de l'amirauté et de onze " anciens patrons, négociants, experts aux affaires maritimes ". L'ancienneté et l'expérience de ces " anciens ", qui ont sans doute mis sac à terre et sont disponibles pour répondre à la requête de l'État, tendent à garantir un savoir et une mémoire des lieux. Si tous les participants sont nommés et si leurs fonctions ont un lien avec le monde de la mer, celles-ci recouvrent des réalités qui resteraient à préciser. Ils sont qualifiés par ailleurs de "prud'hommes ", sans doute pour souligner leur sagesse, car si la pêche est une activité importante à Martigues, une prud'homie n'y est créée qu'en avril 1791. Il est également vrai que cette appellation était parfois en usage dans les confréries de pêcheurs ${ }^{14}$.

Pour " mener la visite du port de Martigues à Port de Bouc ", au cours de la seule journée du 22 mai, ces hommes utilisent " un bâtiment de mer " au type non précisé. Ils quittent l'intérieur de l'étang de Berre, où se situent " les » Martigues - formés depuis 1581 par l'union des bourgs de Jonquières, Ferrière et l'Île - et traversent un plan d'eau d'environ sept kilomètres, dit étang de Martigues, avec le port de Bouc à son extrémité, sur les rives de la Méditerranée.

Lors de ce déplacement ils mentionnent immédiatement la présence des bordigues ou pêcheries fixes, "inféodées à des particuliers " qui ne sont pas nommés ${ }^{15}$. À l'occasion d'une halte à l'embouchure, au port de Bouc, abrité du vent et des dangers extérieurs par une forteresse reconstruite en 1664, les experts justifient leur venue auprès des responsables des lieux

13. BnF, département des manuscrits, 500 Colbert, no 199, fo 207 à 211.

14. BuTI, Gilbert, " Prud'homies de pêche de la France méditerranéenne ", dans CÉRINO, Christophe, Michon, Bernard et SAUNIER, Éric (dir.), La pêche : regards croisés, Rouen, Presses universitaires de Rouen et du Havre, 2017, p. 85-100.

15. MAUNIER, Francis, "Les bourdigues de Martigues à la fin du XVIII" siècle ", Provence historique, t. 14 , fasc. 55,1964 , p. $77-85$. 
par la " lecture de l'arrêt royal ». Ils décrivent sommairement la forme et la taille du port sans en fournir les dimensions, tandis que les sondages réalisés par Étienne Combe indiquent la présence d'un écueil " appelé vulgairement foucard " ou " roche de Foucar ". Une attention particulière est accordée à " l'endroit par lequel les barques et vaisseaux entrent dans le dit port ", car là sont signalées des pierres de taille au fond. La mémoire des " anciens " rappelle :

" quelles y furent emportés la nuit qui précéda le 17 janvier, fête SaintAntoine, de l'année 1636, en laquelle il se leva une grande tempête que les vagues de la mer abattirent la partie de muraille qui fermait la dite forteresse du côté du couchant; quoique la grosseur d'icelle fut d'une cane et demie ${ }^{16}$, l'eau de la mer entra dans le dit port avec une telle violence qu'elle emporta les ruines au-dessous et tout contre ledit rocher; lesquelles ruines il serait avisé de les entourer [bien qu'elles] ne donnent pas grande incommodité aux barques ou navires entrant audit port ".

\section{Figure 2-De Martigues à Port de Bouc (vers 1760) ${ }^{17}$}

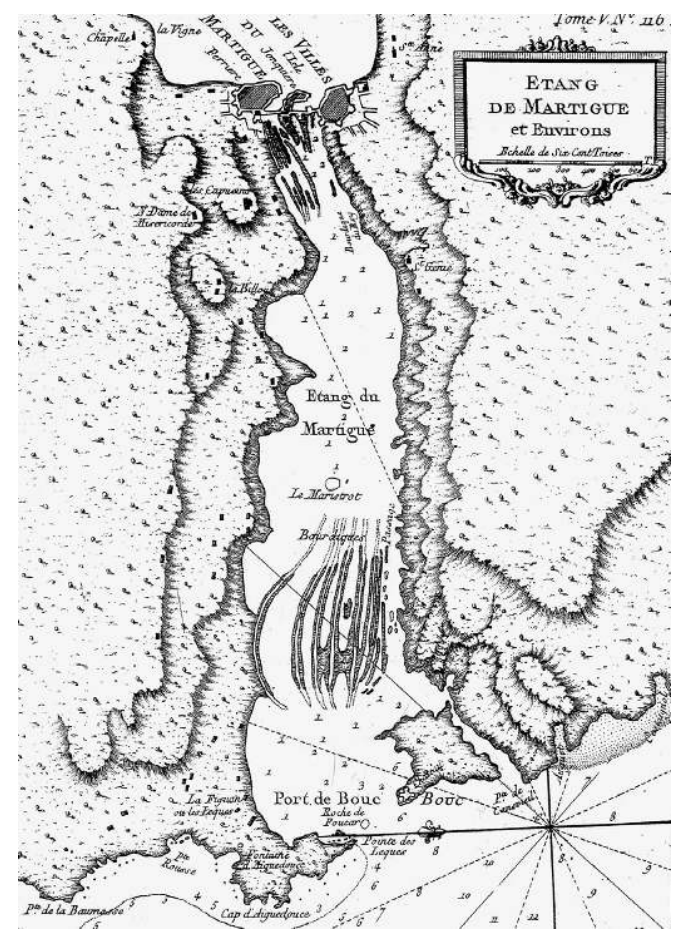

16. Les unités de mesure utilisées appellent quelques précisions; ainsi en est-il de ces " 14 pans qui est une cane à 6 pans la cane de ce pays, revenant à peu près à la toise de France."

17. Belin, Jacques Nicolas, Le Petit Atlas maritime. Recueil de cartes et plans des quatre parties du monde. Vol. v Contenant les costes de France et les places maritimes sur l'Océan et sur la Méditerranée, Paris, 1764. 
L'accessibilité du port étant au cœur de l'enquête, les experts pointent de nouveau les " avancements des bordigues qui incommodent l'usage du port " malgré un arrêt de la cour du parlement de Provence du 12 novembre 1627 limitant l'étendue de ces pêcheries. L'arrêt est demeuré sans exécution " par les suites et oppositions que les propriétaires y ont apportées; les officiers de ce siège n'ayant osé prendre connaissance de ce pour être, la dite cour, saisie de la cause ". Les frictions sont pourtant fréquentes et les procès nombreux, qui se poursuivent jusqu'au milieu du XIX ${ }^{\mathrm{e}}$ siècle, entre les usagers de cet espace maritime et les représentants de la famille Galliffet qui exploitent ce type de pêcheries. La critique est faite mais le nom des Galliffet n'est pas mentionné. La présence d'Alexandre de Galliffet au parlement de Provence (président de la Chambre aux enquêtes) rendelle compte de ce silence dans le rapport écrit relatant l'opération menée sous la conduite de Pierre Romey, avocat au parlement et premier consul?

Les experts poursuivent leur visite à proximité du port de Bouc - " port de Boatier " - qui pouvait recevoir, " cela fait 50 à 60 ans ", des galères, des barques et des polacres, soit des bâtiments de 80 à 150 tonneaux. Or, c'est désormais difficile étant donné " le comblement arrivé, tant par moyen des immondices de la mer que de la boue que les anciens propriétaires desdits bourdigoux y ont jetés " alors que "les jettements de boue étaient autrefois défendus par sentence du 14 août 1689 [sic] à peine de punition corporelle ". Le préjudice est d'autant plus important que " des barques et autres bâtiments de mer servant tant au négoce qu'à la pêche y sont en grand nombre ». Les experts, qui ne font état d'aucun relevé de profondeur, suggèrent de creuser afin " d'y mettre du fond suffisant pour le séjour des vaisseaux et galères ", ce qui ne serait pas difficile à réaliser car "le fond n'est que boue molle aisée à enlever ". La prise en charge et le coût de l'opération ne sont pas abordés, seule la question des droits exigés par les autorités portuaires est rappelée brièvement - " aucun droit autre que le droit d'ancrage appartenant à Monseigneur le duc de Vendôme ". Toutefois, les experts signalent qu'un lieu, " du côté de la Bize ", est dédié à la décharge du lest, ou " sanours qu'on appelle en ce pays ", mais dénoncent aussitôt des vaisseaux ou navires déchargeant de nuit leur lest dans le dit port malgré le garde établi par les consuls pour surveiller. Qui plus est, à la suite de l'arrêt de 1627 relatif aux pêcheries, le commissaire à qui avaient été désignés divers endroits pour servir à la décharge du lest les avait fait " environner de murailles", mais celles-ci ont été rompues, les pierres emportées sans que les officiers de l'amirauté aient osé saisir la cour. C'est sur cette nouvelle mise en cause de l'attitude timorée des représentants de l'amirauté que se termine le procès-verbal. Ne faut-il pas rapprocher cette attitude à l'encontre des représentants de l'administration centrale de la manifestation, autrement plus violente, qui secoue Martigues en 1670, au moment de l'instauration du système des classes de la Marine ${ }^{18}$ ?

18. Pillorget, René, " Une émeute de gens de mer (Martigues, 16 août 1670) ", 93e Congrès national des Sociétés savantes, Paris, 1968, p. 313-324. 
L'état du port de Marseille intéresse doublement le pouvoir central dans la mesure où il doit répondre aux besoins du commerce maritime et à ceux de l'arsenal des galères établi après la remise au pas de la cité à la suite des troubles liés à la Fronde ${ }^{19}$. La lutte contre l'envasement provoqué par les eaux pluviales et la négligence des hommes est ancienne. Au XIII siècle déjà, les statuts de la ville assujettissaient à une taxe les propriétaires qui, faisant réparer leurs navires dans le port, risquaient d'en salir les eaux en y évacuant les déchets ${ }^{20}$.

En 1665, en réponse à la demande royale, la visite du bassin portuaire est organisée à compter du 8 avril et occupe trois jours pour un espace plus réduit que celui inspecté à Martigues. Elle est effectuée en trois temps et débute par la visite du plan d'eau ${ }^{21}$.

\section{Figure 3 - Marseille au milieu du XVII siècle ${ }^{22}$}

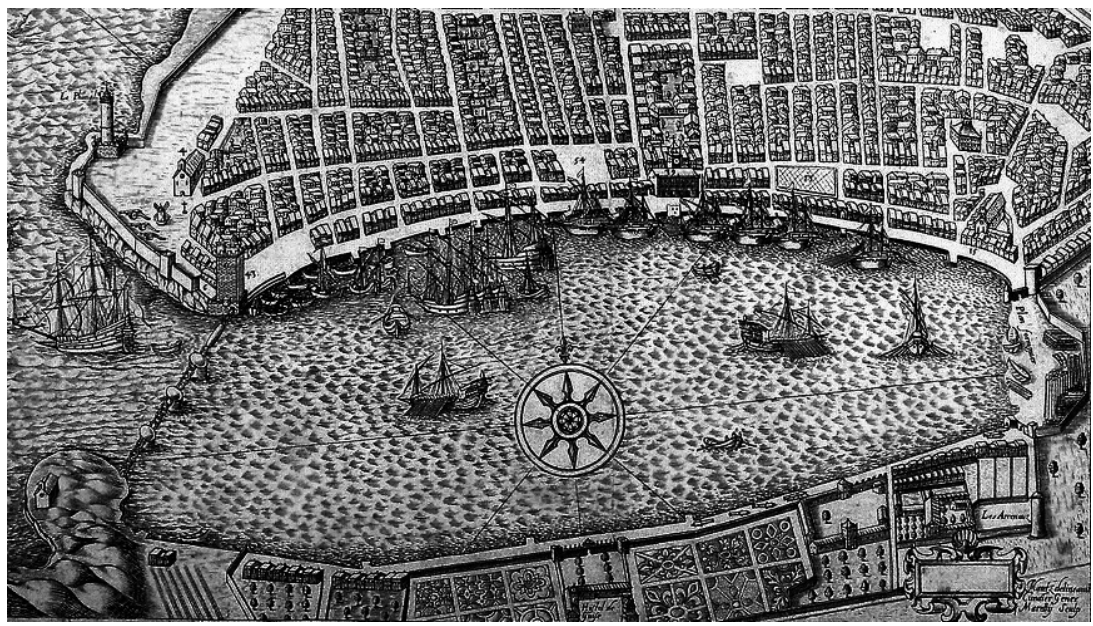

Le lieutenant de l'amirauté (Valbelle), le procureur (Garnier) et le greffier (Bayon) se rendent, à bord d'une petite embarcation, à l'entrée du port, communément appelé " teste de more ", où les attend un homme d'expérience, Louis Soucheiron, " ancien patron de cette dite ville, âgé de 65 ans, auquel ils ont enjoint de sonder ce que la mer a de profondeur". L'itinéraire suivi dans le bassin par ces experts est précisé dans le procès-verbal, avec la mention de points de repères bien identifiables le long des quais et constituant autant de stations : "tête de more [...] Fanal [...]

19. Morineau, Michel, "Flottes de commerce et trafics français en Méditerranée au XVII $^{\mathrm{e}}$ siècle ", XVII siècle, ${ }^{\circ}$ 86-87, 1970, p. 135-171.

20. Arch. mun. de Marseille, Statuta civit. Mass. lib. IV, cap. vI, charte du 18 mai 1243.

21. BnF, département des manuscrits, 500 Colbert, no 199 , fo 179-181.

22. Marseille, plan en élévation (détail) par MARETZ, Jacques et CUNDIER, Louis (vers 1645) d'après La ville figurée, Marseille, Musées de Marseille, 2005, p. 40. 
Chaîne [...] fontaine Saint-Jean [...] maison de Cabriès [...] couvent SaintAugustin [...] quai neuf ".

Sur la rive nord, occupée par les navires marchands et des galères, la profondeur est "suffisante mais inégale [...] il n'y a point de rochers fermes parce que les six engins qui servent à la cure du port s'arrêtent aux endroits les plus faciles...". En revanche, le long de la rive sud, dite rive neuve ou Marquisat, encore peu occupée mais qui doit accueillir l'arsenal, la profondeur est extrêmement faible. Pour Soucheiron, c'est là le résultat de la construction, en 1622, d'un quai à la place du plan incliné : " la mer quand elle est agitée, ne peut pas se purger par le dehors comme elle le faisait autrefois quand ce n'était que plage; il y avait alors le long de la dite plage et dans le dit port un fond beaucoup plus grand qu'il y a à présent ". Soucheiron donne de la force à son témoignage en déclarant avoir vu, de ses propres yeux, l'état de la dite rive avant son aménagement. En ne permettant plus un " effet de chasse " le quai perturberait la circulation des eaux dans le bassin et en favoriserait l'envasement. Le délestage des navires n'intervient donc nullement dans la déposition de cet ancien patron pour rendre compte de la faible profondeur constatée.

Figure 4 - État de la profondeur du port de Marseille en $1665^{23}$

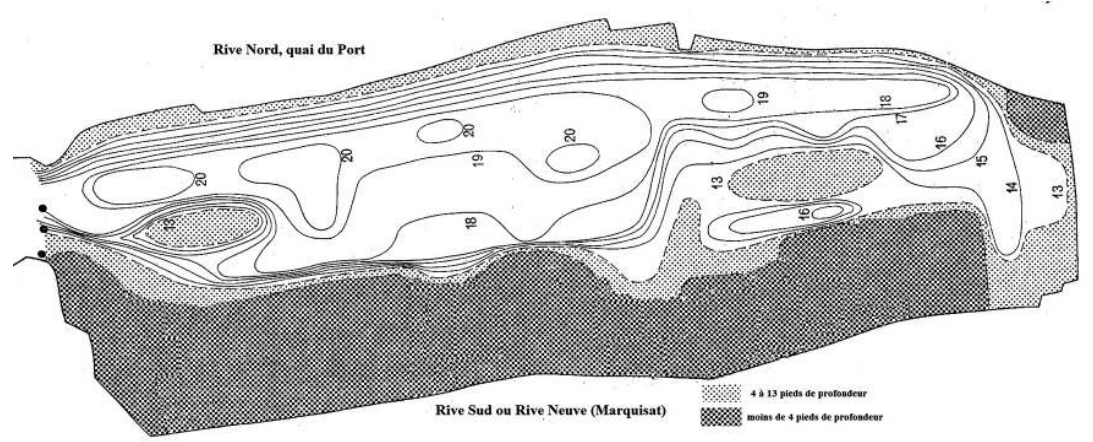

Le lendemain, à 8 heures, mission est confiée chez Valbelle à Pons Reynaud, " professeur aux mathématiques de cette ville ", de mesurer le bassin (longueur, largeur, surface). Les modalités pour ce faire ne sont pas précisées. Les résultats communiqués " à 4 heures après midi » font état :

" d'une étendue du dit port de 76872 toises carrées, de 488 toises de longueur, et dans sa plus grande largeur, qui est à la palissade dite des oursins, 164 toises, et 46 toises un quart à l'embouchure, y compris les piliers

23. D'après RAMBERT, Gaston, Histoire du commerce de Marseille, t. IV, 1599 à 1789, Paris, Plon, 1954, p. 416 et CARRIÈre, Charles, Négociants marseillais au XVIII siècle. Contribution à l'étude des économies maritimes, 2 vol., Marseille, Institut historique de Provence, 1973, p. 161. 
qui soutiennent la chaîne ayant chacun 21 pieds de long, 13 de large relevés par-dessus l'eau de 6 pieds et 2 pouces étant inégalement éloignés l'un de l'autre."

Le 10 avril, Valbelle, toujours en compagnie du procureur Garnier et du greffier Bayon, convoque, dans sa demeure, pour audition quatre experts liés aux affaires maritimes, à savoir un patron (Philip Icard, 80 ans), un nocher (Jacques Nitaud, 60 ans), un marinier (Charles Icard, 45 ans, fils de Philip) et un ancien prud'homme des pêcheurs (Laurent Lombardon, 66 ans) ${ }^{24}$. Après serment, ces "sachants ", aux âges rappelés comme gage d'expérience et de sagesse, relèvent " unanimement et d'un commun accord " trois raisons pour expliquer la situation de la rive neuve. Ils rejoignent en premier lieu les propos de l'ancien patron Louis Soucheiron, auteur des sondages, en mettant en cause le quai neuf qui fait de la "résistance aux ondes de la mer ". Ils critiquent ensuite le curage du port en précisant que les responsables des opérations cherchent les endroits les plus faciles à dégager pour en faire davantage afin de gagner plus, " attendu qu'ils sont payés suivant la plus grande quantité de boue et autres matières qu'ils tirent du port, dix livres la barquade ${ }^{25}$ ". Par ailleurs, "les engins qui travaillent ne sont pas aussi forts qu'ils l'étaient anciennement ${ }^{26}$ ". Les experts mobilisent leur mémoire pour décrire la machine qui était alors employée par un Vénitien, " Jacques Trevisas, décédé depuis environ vingthuit années ", puis par son gendre Claude Ferras, " encore en vie " - ce qui ne peut que renforcer les déclarations des témoins - qui donna avec cet " engin fait à cuillères [...] vingt-deux pieds de profondeur à l'entrée de la chaîne où il n'y en avait pas auparavant quinze ". Enfin, ils mentionnent l'absence de « lieu le long du port, ni d'un côté, ni de l'autre, pour y décharger les lests des vaisseaux et des barques qui abordent ". Dans ces conditions, les usagers déchargent les lests " sur l'un ou l'autre quai, ou dans la mer ", alors qu'en application d'un règlement local, " les intendants, qui sont annuellement désignés par le Conseil de la ville pour assurer la cure du port, ne souffrent pas qu'on jette les lests dans la mer ». Surpris par les gardes des intendants, le contrevenant est condamné au paiement d'une amende. Les experts rappellent qu'il existe bien, pour la cure du port, "le paiement d'un droit de demi pour cent $(0,5 \%)$ sur toutes les marchandises qui entrent en cette ville par mer, pour le compte des étrangers qui ne sont pas de la dite ville, droit arrenté la présente année à 19000 livres ", mais ignorent "si c'est tout pour la cure du dit port ». Le constat établi, ces

24. La prud'homie de pêche de Marseille, la plus ancienne de Provence, est attestée depuis le début du XIV ${ }^{\mathrm{e}}$ siècle, sinon avant, autour de la confrérie de Saint-Pierre.

25. Aucune précision n'est apportée au sujet d'un éventuel usage de ces boues (amendement de terres voisines?)

26. Sur les techniques utilisées pour l'entretien des ports, voir LLINARÈs, Sylviane, "L'entretien des "ports de France" à l'époque moderne. Techniques, mise en œuvre et innovations $\left(\mathrm{XVII}^{\mathrm{e}}\right.$-XVIII ${ }^{\mathrm{e}}$ siècles) ", dans LLINARÈs, Sylviane, ÉGASSE, Benjamin et DANA, Katherine (dir.), De l'estran à la digue. Histoire des aménagements portuaires et littoraux, $X V{ }^{e}-X X^{e}$ siècle, Rennes, Presses universitaires de Rennes, 2018, p. 15-41. 
experts proposent que l'extrémité du quai neuf soit réservée au déchargement des lests; ne signent la déposition que " ceux qui ont su écrire ", à savoir Valbelle, Garnier et Bayon.

À Toulon, l'enquête "soumere " [sommaire] a mobilisé huit experts durant une semaine, du 11 au 17 juin, a donné lieu à un rapport détaillé de 13 folios (soit 26 pages), accompagné de multiples suggestions et précédé du rappel intégral des consignes gouvernementales ${ }^{27}$. Les opérations s'y déroulent en deux temps : d'abord une série d'auditions, puis la visite, avec sondages, des rades - de la petite seulement - et de la darse. Du 11 au 15 juin, six personnes sont entendues, à tour de rôle, dans la maison de Vincent de Ricard, conseiller du roi et lieutenant général de l'amirauté : trois marchands (Pierre Allardon, 66 ans, Antoine Allardon et Gaspard Voire aux âges non indiqués), un maître hache (Jacques Ubac, 68 ans) qui a la " direction du ponton qui sert au creusage du port ", un ancien capitaine (Jacques Gaudy, 70 ans) et un ancien maître d'équipage de l'armée navale (Jean Truffaut, 66 ans), lequel participe également, 48 heures plus tard, à la visite du port.

Outre les deux carcasses de vaisseau du roi qui gênent la circulation à l'entrée de la darse, le dénominateur commun à l'ensemble des dépositions de ces acteurs du monde maritime est le mauvais état du bassin qui résulte de la négligence des usagers et de facteurs naturels. Ainsi, les débris des maisons en construction déposés près du quai sont entraînés dans le port par les pluies, tandis que l'eau du port " est grandement endommagée au moyen de la soude que les savonniers mettent à la rue après avoir fait le savon ". Au reste, le quai étant insuffisant pour décharger et charger le lest, cette opération se fait à l'ouest du bassin, près du " parc et de la muraille ", dans un espace occupé par les navires de guerre et où s'esquisse l'aménagement d'un arsenal ${ }^{28}$. Sans venir jusqu'au quai, « quelques capitaines ou officiers de navire de Sa Majesté jettent le lest de leurs navires dans la rade, au lieu de le faire porter à terre, ce qui a beaucoup contribué à gâter icelle aussi bien que l'embouchure du port ». Mais ce sont surtout les " vases, pierres et alluvions " charriées par deux torrents - le Las, à l'ouest, et l'Eygoutier, à l'est - qui provoquent l'accumulation de dépôts à l'entrée de la darse, près de la chaîne, tandis que " la marée, qui vient de la grande rade, pousse avec violence la vase, les pierres et autres choses que ces torrents emmènent dans l'embouchure du port et qu'ils le comblent ${ }^{29}$ ".

Hormis un meilleur usage de la machine à curer le port, le retrait des carcasses, l'interdiction des dépôts de gravats, la construction de palissades pour empêcher leur jet à la mer, les rejets des savonneries, la création d'un

27. BnF, département des manuscrits, 500 Colbert, nº 199, fo 184 à 197.

28. CROS, Bernard,« L'arsenal de Toulon et la défense de la rade de Louis XIII au début du règne de Louis XIV ", Revue de la Société des Amis du vieux Toulon et de sa région, $\mathrm{n}^{\mathrm{o}} 134,2013$, p. 117-150.

29. La Méditerranée n'ignore pas totalement le phénomène des marées mais celles-ci sont généralement de faible amplitude, de l'ordre de 10 à 20 centimètres à Toulon. 
quai supplémentaire et la construction d'un " pilier, près de la chaîne " pour gêner l'entrée de la vase dans le port, plusieurs propositions sont faites pour détourner les cours des deux torrents responsables de l'envasement. Au vrai, lors de l'inspection réalisée en 1633 par Séguiran, les officiers de l'amirauté avaient déjà signalé ces deux torrents qui charriaient " pierres, graviers et limons remplissant le port et empêchant les grands vaisseaux de s'approcher de la darse, ce qui était d'une grande incommodité pour leur chargement et déchargement ${ }^{30}$ ". L'envoyé de Richelieu s'était rendu sur le site pour procéder "à l'estimation des sommes auxquelles pourrait monter la dépense nécessaire " pour effectuer ces " détours " mais l'opération était restée sans suite.

En 1665, des suggestions d'ordre réglementaire accompagnent également les mesures techniques, notamment le renforcement du contrôle du quai en " commettant des surveillants " car le gardien du port établi par les consuls, en application d'un règlement local, pour veiller au lestage et délestage des bâtiments de mer "sans en exiger aucun droit ", ne suffit plus.

Lors de ces dépositions, une suggestion originale est à mettre à l'actif de Jean Truffaut, combinant à la fois la gestion du lest et les projets d'aménagement portuaire :

"Si Sa Majesté voulait faire un des beaux darcenal [sic] de toute l'Europe, il faudrait allonger celui qui y est à présent [...] et y faire un quai [...] sur lequel le lest desdits vaisseaux pourrait être mis et repris, sans gâter le port; et pour faire son avancement il faudrait obliger tous les bâtiments de mer de jeter le lest dans l'eau qui y est à présent et qui n'étant pas profonde serait bientôt remplie [...] ce ferait espace pour recevoir non seulement le lest, mais tous les canons, et y mettre encore tant bois à fabriquer autant de galères et vaisseaux qui plairait à $S$. M. "

La visite des rades et de la darse est réalisée le 17 juin par six personnes à bord d'une petite embarcation. Aux côtés de François de Ricard (lieutenant particulier), Rouvière (procureur), Gairoard (greffier), deux hommes connaissant bien le plan d'eau ont été engagés pour procéder aux sondages : le pêcheur Pierre Mounier et Jean Truffaut, ancien maître d'équipage de l'armée navale, déjà auditionné et connaisseur de la situation car il a " diverses fois sondé la darse, les petite et grande rades ", sans que les circonstances de ces actions antérieures soient rappelées. Par ailleurs, Vincent de Ricard désigne Jacinthe de Ricard :

" enseigne dans le régiment de Charambert, pour en faire prendre un plan aux fins que Sa Majesté puisse voir plus clairement l'incommodité et dommage que les deux torrents de l'Eygoutier et du Las causent dans la darse et port où ils se déchargent ensemble, les lieux où ils pourront être détournés et mesurer l'incommodité et dommage que quelques navires de sa Majesté, qui sont à fond $[\ldots]$ causent au mouillage des navires. "

30. BuTı, Gilbert, " Henri de Séguiran en visite sur les côtes provençales (1633-1635) ", Revue de l'Académie du Var, t. XIV, 2013, p. 151-155. 
Le patronyme du dessinateur, certes répandu localement, invite toutefois à supposer un lien de parenté (non encore établi) avec le responsable de l'amirauté qui l'a désigné pour cette mission.

La sortie en mer se fait en deux temps, dans la petite rade puis dans la darse. Une attention particulière est portée une fois encore aux effets des deux cours d'eau situés de part et d'autre du port. Jean Truffaut, qui dirige les opérations, signale la présence " de quantité d'herbes et migou qui retiennent le charriage de l'Eygoutier... " et fait état d'un phénomène inexpliqué - sans doute un courant sous-marin mais aux effets difficiles à formaliser - quand il constate que le torrent aurait " déjà rempli toute la darse et la rade s'il n'y avait pas le vallat de Cougourde qui, par certain secret de nature inconnue aux hommes [...] recevant une partie du charriage de l'Eygoutier, ou l'engloutit ou la jette dehors".

L'opération est minutieusement réalisée avec de nombreux sondages en des points privilégiés par la circulation des navires. Le procès-verbal porte mention de localités riveraines voisines (Saint-Mandrier, La Seyne), de fortifications (Grande Tour, tour de Balaguier), de lieux-dits ou repères côtiers (pointe de Brégaillon, Lagoubran, creux de Saint-Georges, la Baumette, pointe du Soullet, île de Millau, Grande Cabane...) et de " bas fonds de vase, comme la Tasse ". Ainsi que le souhaitait Vincent de Ricard, a été réalisé un dessin rehaussé à la gouache pour accompagner le procès-verbal. Relativement précis, malgré quelques maladresses dans la représentation, il fournit les éléments majeurs responsables, selon les témoins, de la dégradation du site portuaire, et localise les rivières qui enserrent le plan d'eau.

Après la visite de la rade, le groupe se rend " dans l'enceinte de la darse " pour effectuer une série de sondages en notant également des repères permettant de localiser les emplacements des mesures effectuées : près de la consigne, à la pointe Rimade, du côté de Saint-Jean, vis-à vis de la place de l'huile, devant la maison de la Ville..., avec une grande irrégularité des résultats, allant de 8 à 40 pans d'eau, avec 17 à 21 pans à l'entrée de la darse, 12 en son centre et 8 à 14 près du quai.

Commis au siège de l'amirauté de Saint-Tropez, en l'absence du lieutenant Honoré Marquezy, Peyronet désigne, en avril 1665, les "bourgeois" Balthazar Martin et Louis Remonde pour "visiter et sonder ", en sa présence, le port et la rade des Canebiers afin de relever, conformément à l'arrêt royal, les " causes de dépérissement du port, les moyens pour le rétablir et les lieux où se font les charges et décharges des lests ${ }^{31}$ ". Ces membres de vieilles familles locales, impliquées dans les affaires maritimes de la place et dans la vie de la cité (en 1665, le premier consul, Honoré Martin, est un parent de Balthazar), procèdent à une triple expertise : celle du golfe de Saint-Tropez, anciennement dit de Grimaud, (dimensions, profondeur, navigabilité, ancrage), puis celle de la rade foraine des Canebiers (taille, profondeur, abri) et enfin, celle du port, dominé par une citadelle,

31. Ibid., fo 213 à 217. 
avec deux quais étroits "bâtis dans l'eau " bordés de maisons, protégé par une digue de grosses pierres mais qui nécessiterait d'être prolongée pour pouvoir " contenir tous les navires et galères de l'armée navale de S. M. ".

Voulant vérifier l'état dans lequel était " anciennement " la profondeur du port - sans référence temporelle - les responsables de l'opération consultent, sous serment mais sans indiquer leurs identités, les "plus anciens bourgeois et marchands, maîtres de navires, pilotes et matelots ". Selon ces derniers, " la profondeur de ce port, aussi bien proche des maisons que des quais, était partout telle qu'à son embouchure, c'est-à-dire de 18 à 25 pans d'eau [soit 5,5 à 7,5 mètres], et par conséquent capable de contenir bon nombre des plus grands navires avec les plus grands chargements en toute sûreté ". Et " pour preuve " de l'état des lieux dressé par ces porteurs de mémoire, les consuls modernes ont " exhibé leurs livres cadastres depuis l'année $1600^{32}$ ". La preuve écrite constitue en l'occurrence un élément majeur, sinon irréfutable, du " dépérissement " du port lié avant tout aux conséquences de la guerre contre l'Espagne, aux pertes de navires à la suite de " mauvais temps " et aux " pirateries continuelles des Turcs d'Afrique ". Les difficultés économiques associées à ces événements auraient conduit les habitants appauvris à s'accommoder d'un bassin dégradé mais suffisant pour de modestes caboteurs "n'exigeant pas un plus grand fond qu'il y a à présent dans le port ${ }^{33}$ ". La présence de "bancs de sable mouvant et de boues ", entraînés par le ruissellement des eaux pluviales, et l'accumulation de lest dans un bassin non entretenu expliqueraient son mauvais état. En réponse à une allusion faite par l'arrêt de 1665 - concernant des officiers qui auraient " négligé une chose si importante au service de sa Majesté " - le responsable de l'amirauté écarte toute responsabilité "des officiers précédents en ce siège [...] puisqu'ils n'en étaient pas chargés et qu'il n'a jamais été levé aucun droit pour cet effet, ni même pour l'entretien du port, si ce n'est un droit d'ancrage que le seigneur du lieu fait exiger. " Pour faire face à cette situation, les curage et creusement du bassin sont nécessaires " par un ponton ainsi que l'on a coutume de creuser les ports de Marseille et de Toulon ". Mais se pose aussitôt la question du financement des travaux car les habitants sont " toujours plus surchargés d'impositions extraordinaires... ". Le commis préconise surtout des mesures préventives en suggérant que le lest soit désormais :

" chargé aux rivages du port, proche des maisons qui sont dans l'enclos de celui-ci, pour éviter, par ce moyen, que les eaux pluviales du lieu n'emportent toujours plus de sable et de boue, qui sont sur les dits rivages, dans le milieu du port [...] et en ce qui est des décharges des lests, nous avons ordonné qu'il sera fait cris publics à son de trompe et placards affichés par tous les lieux accoutumés et à notre greffe, qu'il appartiendra de faire la décharge des lests derrière la maison du Jeu de paume et la Chapelle SaintElme, bâties sur le milieu du quai entre la Tour Saint-Torpès [sic] et la cha-

32. Livres cadastres qui peuvent être consultés aux Arch. mun. de Saint-Tropez, CC 12 à 16 .

33. La désintégration de la flotte de commerce a été relevée dans l'enquête de 1664. 
pelle Notre-Dame, soit sur la plage d'où nulle sorte de vent ne les peut retirer, avec inhibitions et défenses de les faire ailleurs en aucun autre endroit, ni de renverser leurs lests sur les quais, ni les faire entrer dans le port..."

C'est en tenant compte de la configuration des lieux, de la direction des vents dominants, de l'encombrement des quais et des usages des patrons de navire qu'a été prise cette décision. Pour une meilleure intelligence des choix effectués, le responsable de l'amirauté a fait faire "un plan et vue figurée du port de St-Torpès et du premier rang des maisons qui sont au bord de la mer [...] où sont marqués les lieux où les charges et décharges des lests doivent être faites".

\section{Figure 6 - Le port de Saint-Tropez en $1665^{34}$}

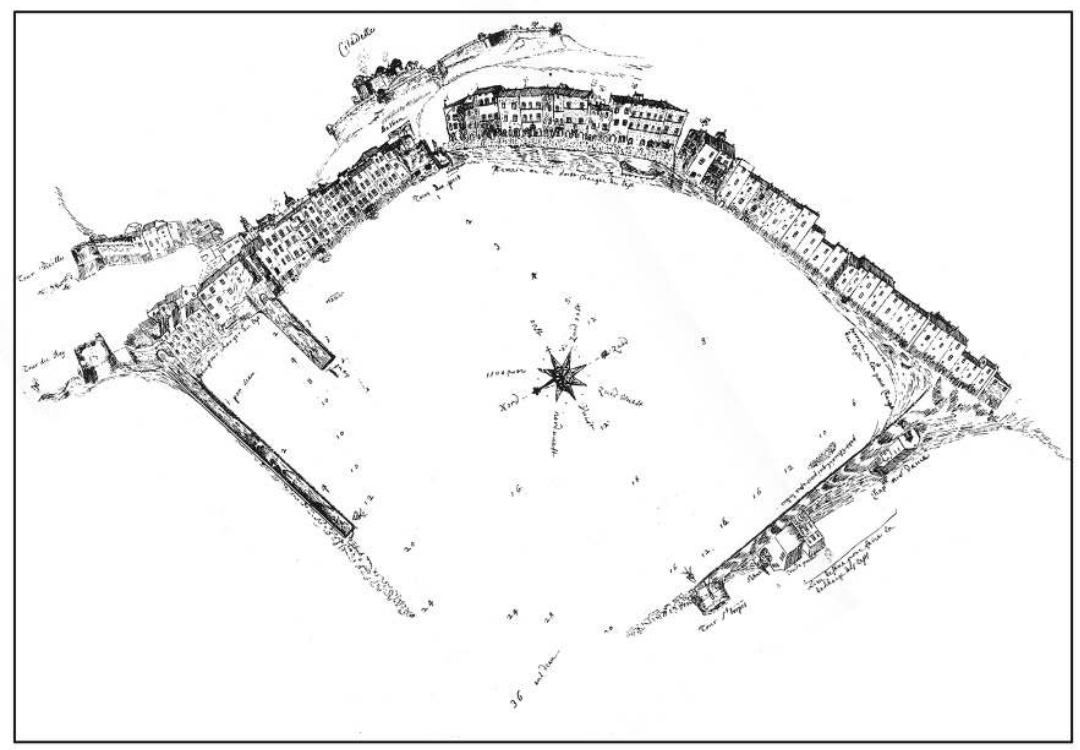

Cette représentation panoramique d'une grande précision, qui suit une technique en usage dans les ports de la rivière de Gênes familiers aux marins tropéziens, porte la mention des profondeurs relevées lors des sondages mais non rapportées dans le procès-verbal ${ }^{35}$. Au reste, attentif aux causes du "dépérissement " du port et soucieux de proposer des moyens de " rétablir " son activité, conformément à la demande de l'arrêt de 1665, le commis Peyronet conclut le procès-verbal en rappelant combien ce port,

34. BnF, département des manuscrits, 500 Colbert, nº 199, fo 213 à 217 et Le port de SaintTropez en 1665. Extrait du greffe de l'amirauté de Saint-Tropez. Bibliothèque du musée de la Marine, PH $36524 \mathrm{~V}$.

35. Calcagno, Paolo, Savona, porto di Piemonte. L'economia della città e del suo territorio dal quattrocento alla granda guerra, Novi Ligure, Città del silenzio, 2013, p. 257 (" Disegno della darsena di Savona ", 1597 et 1620) 
situé à proximité de nombreuses ressources, pourrait être utile au service du roi, et particulièrement à sa marine (bois, chanvre, vivres...).

\section{D’inégales suites : jeux et enjeu de pouvoirs}

Les résultats de ces enquêtes sont difficiles à apprécier, mais le poids économique du port et la politique maritime de l'État royal semblent des facteurs déterminants dans l'application ou non des propositions faites à l'issue de l'enquête de 1665.

À Martigues, où semble se percevoir une tension entre les responsables locaux et le pouvoir central, les quelques suggestions exprimées n'ont guère été suivies d'effets. Au vrai, à la fin du XVII ${ }^{\mathrm{e}}$ siècle, le port entre dans une période de déclin. Les conséquences des guerres, puis celles des contagions, de la croissance commerciale de Marseille et de l'augmentation de la portée des navires de haute mer confirment, au siècle suivant, l'assoupissement des activités, alors que d'interminables procès continuent d'opposer des caboteurs et pêcheurs à la famille Galliffet qui exploite les bordigues considérées par les premiers comme une entrave à la circulation sans que la question du lest soit relevée par les usagers du port ${ }^{36}$.

Après la période de "dépérissement " du port de Saint-Tropez, signalée à Colbert en 1665, le regain d'activité qui s'esquisse dans les années 1680, invite à réaliser l'indispensable travail " pour rétablir le négoce et le trafic maritime ${ }^{37}$ ". L'entretien du bassin prend une acuité nouvelle. En 1688, Nicolas Angallier, entrepreneur de Toulon, dirige les opérations de nettoiement du port en utilisant du matériel provenant de l'arsenal : un ponton offert par Nicolas de Vauvré, Intendant général des mers du Levant, et deux bateaux percés pour transporter la saffre ou vase, technique éprouvée à Marseille et Toulon. Les administrateurs de la ville tentent de pallier le manque de matériel disponible en finançant la construction d'une bete ou tone pour servir au creusement du port mais sans succès semble-t-il car ils confient, en 1700, une nouvelle opération de creusage à Louis Daniel, de La Seyne ${ }^{38}$. La menace d'envasement s'avère constante sans un entretien incessant, et malgré les interdictions et les cinquante livres d'amende pour les contrevenants, il faudra, à diverses reprises, comme en 1725, " défendre aux patrons d'entreposer leur lest au môle du Levant ${ }^{39}$ ". En 1738, un officier venu réaliser à Saint-Tropez un " plan en sonde du port » alerte le secrétaire d'État de la Marine car " les galères du Roi ne peuvent plus entrer dans le port. Comme depuis plus de trente ans il n'y a plus de machine à creuser, il s'est rempli, et si on le néglige encore quelques années, nul bâtiment n'y

36. CARRIÈRE, Charles, "Y-a-t-il eu un XVIII ${ }^{\mathrm{e}}$ siècle à Martigues? ", Provence historique, t. 14, fasc. 55,1964 , p. 53-68.

37. Arch. mun. de Saint-Tropez, BB 10. Délibérations communales, 1687. Voir BuTI, Gilbert, Les Chemins de la mer. Un petit port méditerranéen : Saint-Tropez (XVII -XVIII siècles), Rennes, Presses universitaires de Rennes, 2010, p. 93-106.

38. Arch. dép. du Var, 3E 24-136, 2 mai 1700.

39. Arch. mun. de Saint-Tropez, BB 11, 1725. 
pourra aborder [...] quand il sera creusé il sera aussi sûr que les ports et darses de Toulon et Marseille ${ }^{40}$ ". Le retrait des carcasses de galères espagnoles, calcinées dans le port au début de la guerre de succession d'Autriche, est l'occasion de son nettoiement en 1748 par un entrepreneur toulonnais ${ }^{41}$. Des sondes réalisées en 1756 montrent la nécessité de renouveler le curage du port et servent de support pour envisager son creusage en 1767, avec l'aide de la logistique toulonnaise, comme c'est également le cas dans d'autres petits ports - Cassis en 1772 - aux moyens techniques limités. C'est la régularité des travaux qui est fondamentale car l'entretien épisodique ne saurait régler les problèmes d'engorgement d'un bassin qui continue à recevoir, nuitamment, le lest indésirable...

Des rapports établis au début du XIX ${ }^{\mathrm{e}}$ siècle par des représentants des Ponts et Chaussées font encore mention de l'urgente nécessité de procéder à l'entretien du bassin, ne serait-ce que pour répondre aux exigences des lancements de navires, et de poursuivre les patrons indélicats ${ }^{42}$. Ces opérations sont toujours longues et onéreuses, voire sources de tensions entre les usagers du port et ses administrateurs ${ }^{43}$.

L'État royal a été sensible aux suggestions faites en 1665 par les auteurs du procès-verbal toulonnais. Sa volonté désormais clairement affirmée de développer sur le site de Toulon un grand arsenal rejoint la préoccupation locale en faisant procéder au détournement des cours du Las et de l'Eygoutier $^{44}$. Les travaux commencés en 1679 sont terminés dès 1681, tandis que le creusement d'une nouvelle darse modifie profondément l'aménagement de la petite rade ${ }^{45}$. Par ailleurs, l'arsenal est doté d'un matériel et d'un personnel chargé de l'entretien permanent des bassins d'un port qui occupe désormais un rôle stratégique de premier plan sur la Méditerranée ${ }^{46}$.

40. Service historique de la Défense, Toulon, Art. 8. Section 1. Carton 1 (1694-1820). Mémoire sur le port de Saint-Tropez (1738).

41. Arch. dép. du Var, 3E 24-148, 15 mai 1748. Les carcasses sont brisées, les chaloupes, agrès et arbres emportés à Toulon par l'entrepreneur Joseph Barralier.

42. Arch. dép. du Var, 4S 16. Travaux publics et transports, trafic maritime : statistiques et tableaux. Voir également PAVLIDIS, Laurent, " La construction navale traditionnelle en France méditerranéenne de la fin du XVIII ${ }^{\mathrm{e}}$ siècle à la fin du XIX ${ }^{\mathrm{e}}$ siècle ", thèse inédite, AixMarseille Université, 2012.

43. BuTI, Gilbert, "Aménagements portuaires et littoraux en Provence (xvII -milieu XIXe siècle) ", dans Llinarès, Sylviane, ÉGASSE, Benjamin et DANa, Katherine, De l'estran..., op. cit., p. 225-240.

44. CROS, Bernard, « Toulon, arsenal de Louis XIV, 1660-1701 » inédit, en dépôt au Service historique de la Défense, Toulon, 1992; Arch. nat., Mar., D2-44. Mémoire sur l'ordre qu'on observe dans l'arsenal de Toulon.

45. Un semblable détournement du cours de torrents côtiers a été réalisé, avec le soutien technique de l'État, à La Ciotat en 1782-1783. Voir Arch. nat., Mar., D2-50. Matériel et travaux hydrauliques. La Ciotat.

46. Joseph Vernet ne manque pas de représenter la machine à curer en action dans la vieille darse de Toulon : Le port vieux de Toulon du côté du magasin aux vivres, 1756. Une telle machine est également présente à Marseille à l'Intérieur du port de Marseille vu $d u$ pavillon de l'horloge du Parc (1754). 
La coïncidence entre les intérêts de la ville et ceux de l'État se retrouve un temps et dans une certaine mesure à Marseille où l'État intervient à la suite du procès-verbal adressé en 1665. Nicolas Arnoul, nouvel intendant des galères et proche de Colbert, qui est nommé conjointement avec les deux échevins pour administrer la cure, augmente aussitôt le nombre de machines, fait accélérer les cadences d'extraction de la boue et diminue le prix des opérations. En janvier 1672, il annonce à son ministre de tutelle qu'il a fait déblayer " cinq canaux pour aller à la rive sud " et lui indique " qu'hier, 11 janvier 1672, il y passa un vaisseau malouin où il y avait plus de 100 ans qu'il en était passé! " Un plan du port établi en 1673 et recueilli trois ans plus tard par Seignelay, montre ce travail accompli pour accéder au quai de Rive Neuve.

Figure 8 - Le port de Marseille en $1673^{47}$

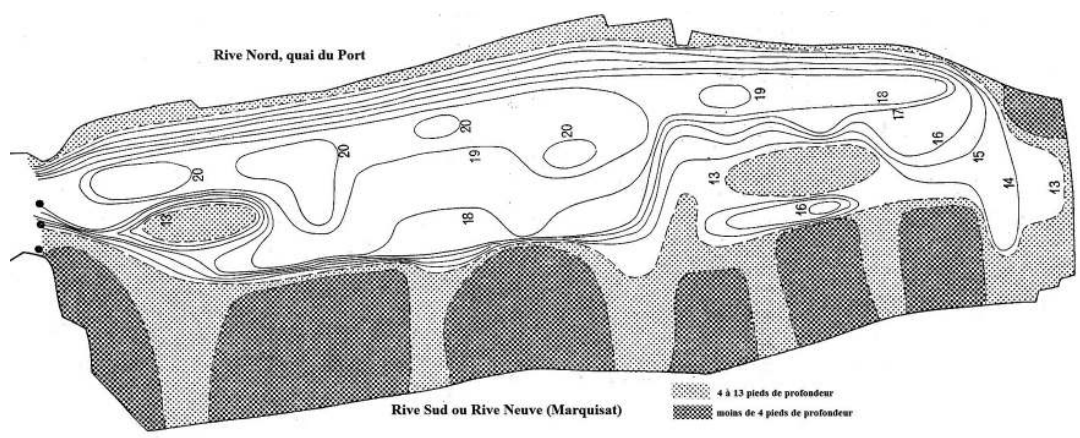

Mais la forte croissance du trafic maritime que connaît Marseille au XVIII ${ }^{\mathrm{e}}$ siècle, l'encombrement du bassin du Lacydon par des navires venus de toute l'Europe, les rejets dans le port d'immondices d'une ville en plein développement et ceux d'activités préindustrielles établies sur la rive sud, notamment après le départ des galères pour Toulon (1748), nécessitent d'incessantes opérations de déblaiement et de creusement. La question de l'entretien du bassin ne se limite pas au seul contrôle de l'État par les représentants de l'amirauté. Elle revient régulièrement, tout au long des décennies, dans les relations entre la Ville, qui est chargée du service, et la Chambre de commerce qui doit pourvoir à la dépense ${ }^{48}$. La situation devient plus claire après 1776 lorsque la Chambre consulaire, toujours responsable du financement, reçoit également la direction des travaux. Ce n'est qu'en 1783 que la machine de Morainville permet un dégagement efficace et rapide, en menant de front les deux tâches complémentaires : le creusement et le déblaiement. Il y avait alors urgence car des bâtiments

47. D'après RAMBERT, Gaston, Histoire du commerce..., op. cit., p. 416 et CARRIÈRE, Charles, Négociants marseillais..., op. cit., p. 161.

48. CARRIÈRE, Charles, op. cit., p. 159-162. 
s'échouaient dans une partie du bassin ayant seulement 8 à 9 pieds de fond. En 1791, le bassin peut être utilisé dans toute son étendue avec une bonne profondeur. Sous réserve d'une surveillance incessante...

Les enquêtes royales, qui contribuent à l'affirmation de l'État sur les rivages et marquent une étape dans l'accroissement de l'efficacité administrative, visent plusieurs objectifs complémentaires : maintien de l'ordre royal (police, justice et armée), connaissance du potentiel militaire (flottes, hommes, matériaux), surveillance des gens de mer (circulation, services), lutte contre les abus (pêches, contrebande, forbans), contrôle des ressources fiscales (taxes, impôts, droits des seigneuries maritimes) et de l'outillage portuaire (accessibilité, entretien, sécurisation).

En souhaitant faire le point sur la situation des ports et havres du royaume, l'enquête de 1665 fournit au pouvoir central un tableau d'une grande diversité. Les réponses apportées à un même questionnaire vont parfois au-delà des informations demandées et portent la marque de contextes locaux, qu'il s'agisse de tensions, comme à Martigues, ou de projets en cours comme à Toulon. Le déroulement de la procédure passe quelquefois sous silence certaines décisions, notamment au sujet des experts ou " sachants " consultés pour leurs compétences, talents et expériences, mais on ignore, tout au moins dans les cas étudiés, les raisons personnelles des choix effectués par les autorités locales. Des recherches complémentaires se heurtent pour l'heure à un déficit documentaire.

La démarche de Colbert éclaire une phase essentielle des enquêtes littorales à savoir le passage de la collecte d'informations à la mise au point de directives d'application, où se mêlent intégration de données anciennes et innovations. Même si elle n'était sans doute pas dans les intentions originelles de ses promoteurs, la consultation des amirautés et par la suite celle d'autres institutions (bureau du service des classes) pour obtenir des renseignements ébauchent un système permanent d'information. Cette enquête, qui a reçu des compléments, a été suivie de mesures locales et prolongée par des textes de plus large portée. Ainsi, l'arrêt du Conseil du 29 mars 1670 réglemente, en dix articles, la manière de procéder au lestage et délestage des grands vaisseaux, et condamne les contrevenants à des amendes, voire à des peines corporelles ${ }^{49}$. Mais c'est l'ordonnance de 1681 qui apporte des éléments majeurs au sujet de la police des ports en synthétisant, dans une large mesure, les éléments recueillis en 1665. Ce texte, qui fournit un cadre général sans exclure les règlements locaux, commence par la question des dépôts sauvages de toutes sortes, se poursuit en enjoignant aux municipalités de placer des grilles métalliques aux égouts qui se déversent dans les bassins portuaires et s'arrête finalement mais longue-

49. Valin, René Josué, Nouveau commentaire sur l'ordonnance de la marine du mois d'août 1681, où se trouve la conférence des anciennes ordonnances..., 2 vol., La Rochelle, J. Legier, 1766, vol. 1, p. 509 (" Du lestage et délestage "). 
ment sur la question du lest. Ainsi, le maître de quai doit visiter chaque mois les chenaux utilisés par les navires, particulièrement dans les ports de guerre. En faisant leur rapport aux officiers de l'amirauté les capitaines sont contraints de déclarer la quantité de lest qu'ils ont à bord avec visite des navires par les dits officiers pour vérification. Des sanctions sont prévues contre ceux qui jettent leur lest dans les ports (amende puis confiscation du bâtiment). Les échevins des villes et communautés doivent désigner, comme d'aucuns le faisaient avant 1681, des lieux destinés à recevoir, en plein jour, le lest de telle manière qu'il ne soit pas emporté par les flots. Les surveillances et contrôles visent tous les ports et havres du royaume, mais la répétition des règlements, y compris à l'échelle locale, laisse entrevoir leur inégal respect.

Un suivi des sentences rendues à l'encontre des contrevenants et l'évolution des sanctions prises ou envisagées alors que progresse, au cours des décennies suivantes, la portée ou tonnage des navires, permettraient d'appréhender l'évolution de la question du lestage dans la vie portuaire. Par ailleurs, il serait tout aussi intéressant de pointer, sinon d'évaluer, la part prise par le lest " permanent ", celui qui reste, en vrac ou non, en fond de cale (pièces d'artillerie), et par celui que Valin qualifie de « lest utile au public ", comme ces pierres ou sable à bâtir, voire ces cendres destinées aux savonneries ${ }^{50}$. En outre, le lest des navires, qui vont sur toutes les mers du monde au cours de cette première mondialisation des échanges, n'a-t-il pas, au même titre que de nos jours les eaux de ballast, contribué à la diffusion dans les bassins portuaires de micro-organismes et espèces invasives non-indigènes ${ }^{51}$ ?

50. Valin, René Josué, Ibid., p. 518. Sur la variété de lest, son possible réemploi ou sa vente, voir pour la période ancienne NANTET, Emmanuel, Phortia. Le tonnage des navires de commerce en Méditerranée du VIII siècle av. l'ère chrétienne au VII siècle de l'ère chrétienne, Rennes, Presses universitaires de Rennes, 2016, p. 201-205.

51. Masson, Daniel, Courtols, Olivier, MASson, Nadine, Guesdon, Stéphane, Rocher, Grégory et MARGAT, Sylvie, "Évaluation du risque d'introduction d'espèces indésirables par l'intermédiaire des eaux de ballast des navires ", Archimer-Ifremer, 1999 et 2000; Direction des Affaires maritimes, Guide pour l'évaluation des risques d'introduction d'espèces non indigènes par les eaux de ballast, Paris, Ministère de l'écologie, du développement durable et de l'énergie, 2014; BuTı, Gilbert et CABANTOus, Alain, De Charybde en Scylla. Risques, périls et fortunes de mer du XVI siècle à nos jours, Paris, Belin, 2018, p. 235-236. 


\section{RÉSUMÉ}

De grandes enquêtes administratives ont été réalisées en France au $\mathrm{XVI}^{\mathrm{e}}$ siècle. Celle qui a été faite en 1665 à la demande de Colbert a pour objet la connaissance de l'état des ports du royaume. Elle pose la question du rôle du délestage des navires dans la dégradation des bassins portuaires. Colbert a sollicité les responsables des amirautés qui ont fait appel à des experts choisis parmi les usagers des ports. L'examen des réponses faites dans quatre ports provençaux (Martigues, Marseille, Toulon et Saint-Tropez) permet d'observer la procédure suivie. L'analyse des procès-verbaux montre la complexité et la diversité des réponses apportées. Celles-ci contiennent des suggestions pour améliorer les situations locales. Certaines ont été suivies d'applications, d'autres ignorées. Outre l'encombrement des plans d'eau, l'emploi du lest pose des questions d'ordre économique et environnemental.

\section{ABSTRACT}

Large administrative inquiries were undertaken in seventeenth-century France. In 1665, at the request of Colbert, on such inquiry sought to determine the state of the kingdom's ports. It raised the question of the role of the unballasting of ships in the degradation of the harbour basins. Colbert worked with the officers of the admiralties who called upon experts chosen among the users of the ports. The examination of the answers given in four ports of Provence (Martigues, Marseilles, Toulon and Saint-Tropez) reveals the followed procedure. The analysis of reports shows the complexity and the diversity of the answers. These contain some suggestions to improve local situations. Some were followed by practical changes, others ignored. Besides the congestion of harbour, the use of ballast poses economic and environmental questions. 
Figure 5 - Le port et la rade de Toulon en 1665

(BnF, département des manuscrits, 500 Colbert, $n^{\circ} 199, f^{\circ} 197$ )

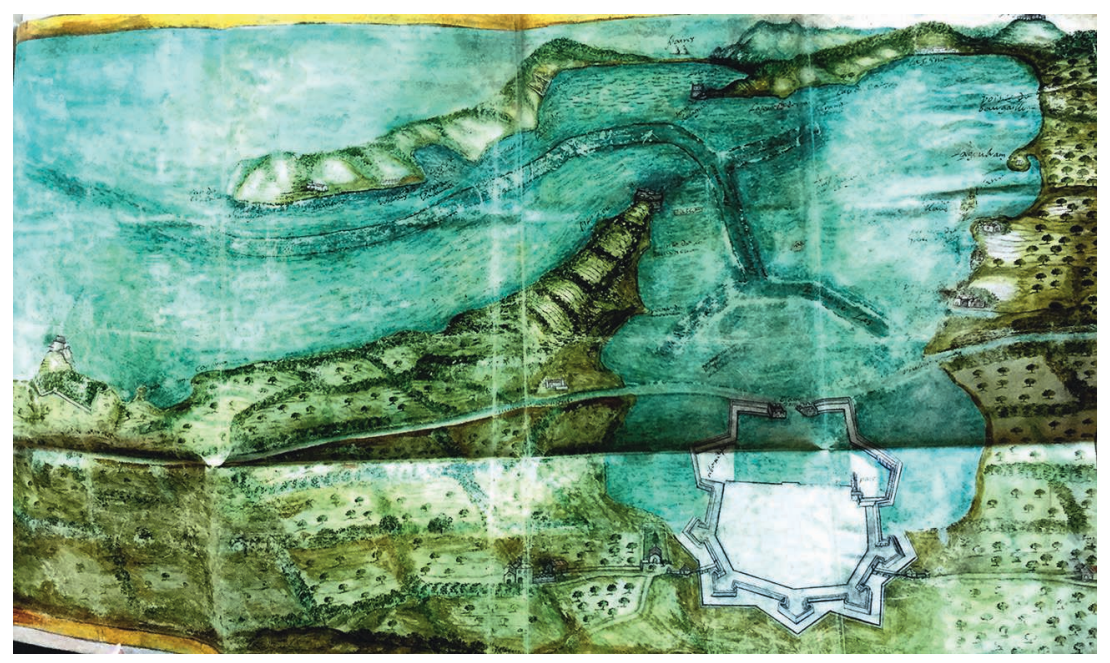


Figure 7 - Plan du port de Saint-Tropez pour servir au projet de son creusage (1767) (Arch. nat., H 1 1262. Port de Saint-Tropez, 1744-1777. Mémoire sur la nécessité de creuser le port et devis estimatif, 24 mars 1767. Dessin de Milet de Monville)

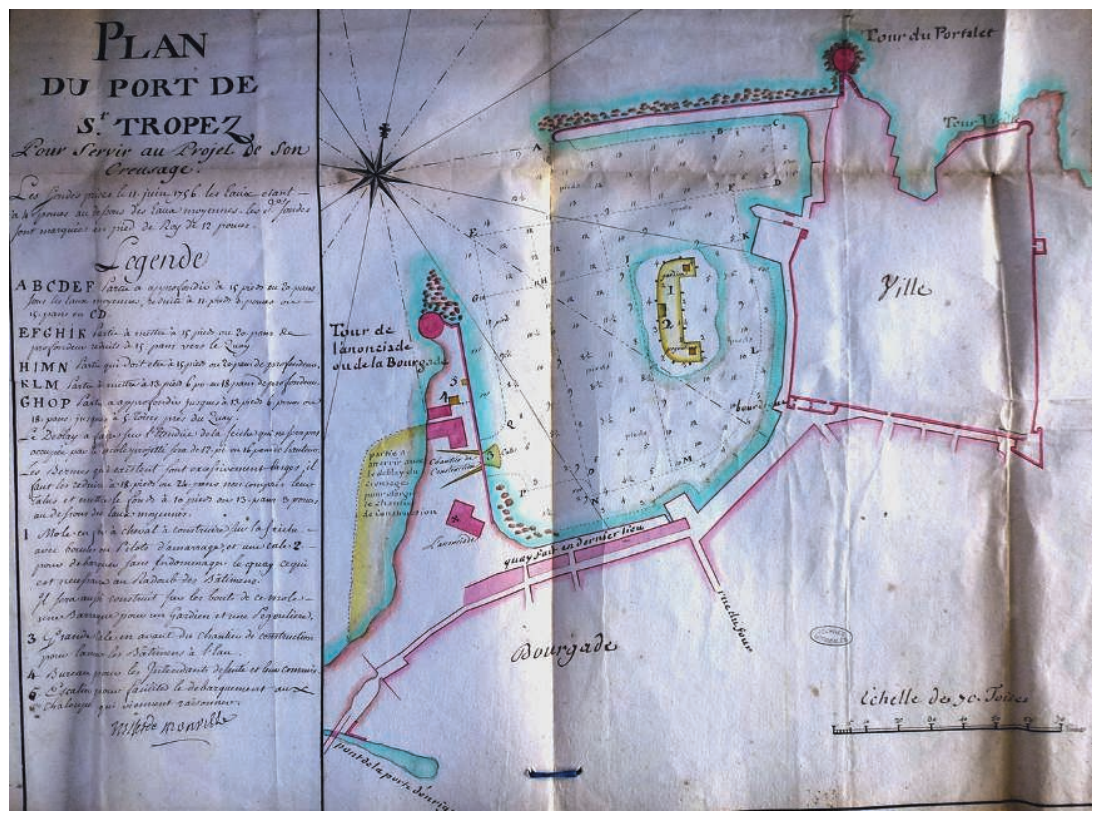

